\title{
Switching Surge Reduction of A Bi-Directional Dual Active Bridge DC-DC Converter with A Digital Operation
}

\author{
Mika Takasaki*, Yoichi Ishizuka*, Tamotsu Ninomiya*, Yutaka Furukawa** \\ and Toshiro Hirose*** \\ Nagasaki University*, Koga System Works** \\ and Nishimu Electronics Industries Co.,Ltd.*** \\ 1-14 Bunkyo-machi, Nagasaki-shi* \\ Nagasaki, Japan* \\ Tel.: $+81 /(95)-819-2556$ \\ Fax: $+81 /(95)-819-2556$ \\ E-Mail: isy2@nagasaki-u.ac.jp
}

\section{Acknowledgements}

The authors would like to thank Mr. Shohei Iwasaki, technical staff of Nagasaki University, for the various technical assistance.

\section{Keywords}

«Converter circuit», «DC power supply», «DSP», «IGBT»

\begin{abstract}
Recently, the bi-directional dc-dc converter has been focused on because of the huge demand for diversification of power supply network including battery. The dual active bridge (DAB) dc-dc converter is one of the most popular circuits for bi-directional applications because of its simple structure. However, power efficiency at light load is the intrinsic problem of a bi-directional DAB DC-DC converter. In this paper, the simple solution with digital operation for the problem is proposed and experiments are performed with $1 \mathrm{~kW}$ system. This method can reduce a switching surge without other circuits such as snubber and improve power efficiency at light load. Therefore it can reduce loss of switching surge, and improve power efficiency. From the results, 37\% maximum power efficiency improvement at light load is confirmed. Furthermore, this method is capable for control in the conventional method in the heavy load range. Consequently, it is possible to reduce the switching surge and realize high power efficiency in a wide load range.
\end{abstract}

\section{Introduction}

Recently, the bi-directional dc-dc converter has been focused on because of the huge demand for diversification of power supply network including battery. The DAB dc-dc converter is one of the most popular circuits for bi-directional applications because of its simple structure [1-5]. However, a switching surge and power efficiency at light load condition is the intrinsic problem [4].

Some research have been done to solve the problem, for instance, use of resonant type converter with snubber circuit [1], silicon carbide ( $\mathrm{SiC})$ power device and new magnetic materials [2], Quasi-ZCS operation with LC filter [3], and converter linked through superposition in additive polarity in series[4].

This paper proposes a simple solution for power efficiency improvement with digital operation. This method can improve power efficiency due to reduce a switching loss without adding other circuits such as snubber circuit. 


\section{BASIC OPERATION OF A DAB DC-DC CONVERTER}

Figure 1 shows the circuit schematic of the conventional DAB dc-dc converter. Figure 2 shows conventional operating waveform [5]. In the conventional operation, the output power is operated by the phase-shift shown as $\varphi$ between the primary voltage $v_{\mathrm{P}}$ and secondary voltage $v_{\mathrm{S}}$ of transformer.

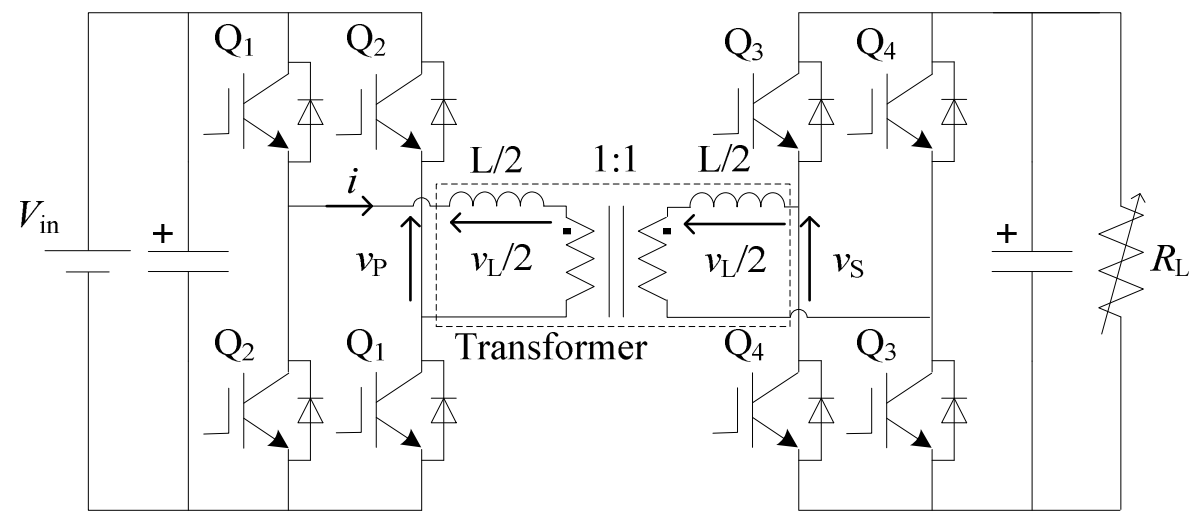

Fig. 1: The circuit schematic of DAB dc-dc converter

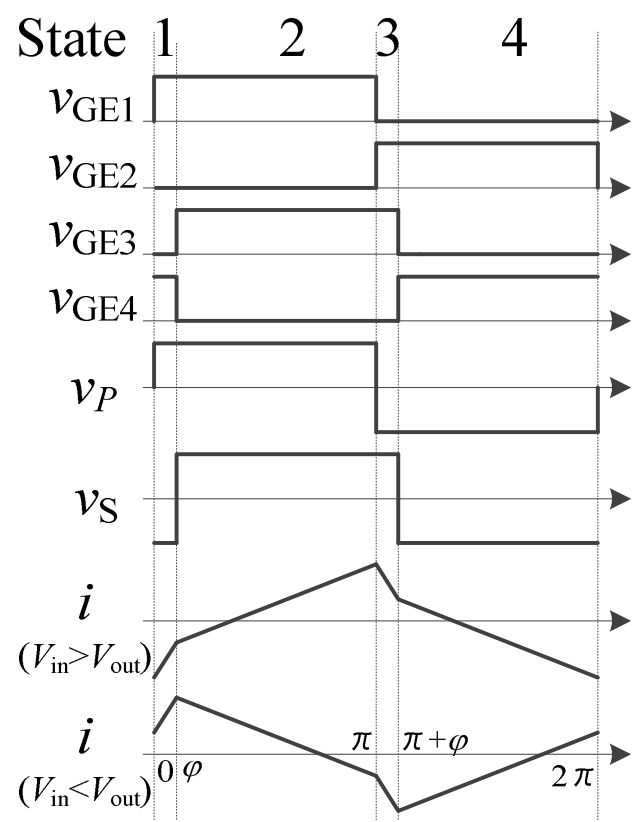

(a) light load

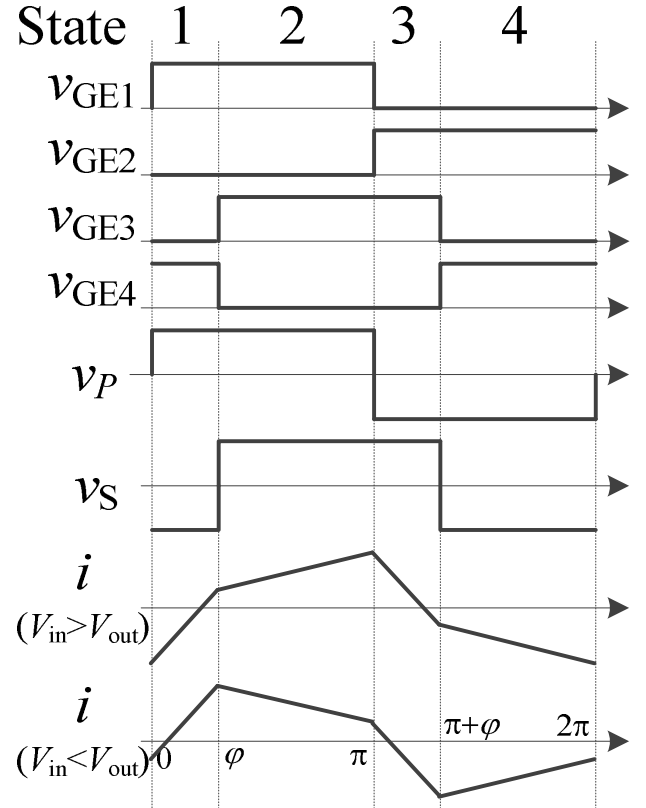

(b) heavy load

Fig. 2: Conventional operating waveform: (a) light load; (b) heavy load

Figure 3 shows the phasor diagram. $V_{\mathrm{P}}, V_{\mathrm{S}}, V_{\mathrm{L}}$, and $I$ are phasor symbols for $v_{\mathrm{P}}, v_{\mathrm{S}}, v_{\mathrm{L}}, i$, respectively. When $V_{\mathrm{S}}$ is leading in phase than $V_{\mathrm{P}}$, converter is operated in power running mode (Fig. 3 (a) and (b)), and when $V_{\mathrm{S}}$ is lagging than $V_{\mathrm{P}}$, it is operated in power regenerative mode (Fig. 3 (c)).

The output power Po can be obtained as

$$
P_{o}=\frac{V_{\text {in }} V_{\text {out }}}{\omega L} \varphi\left(1-\frac{\varphi}{\pi}\right) .
$$

The output power can be controlled with the phase difference $\varphi$. The waveform of the current $i$ is changed by the load condition. In this paper, current $i$ crossed the zero line in the State 2 is defined as a light load, and current $i$ crossed the zero line in the State 1 is defined as a heavy load as shown in Fig. 2. 

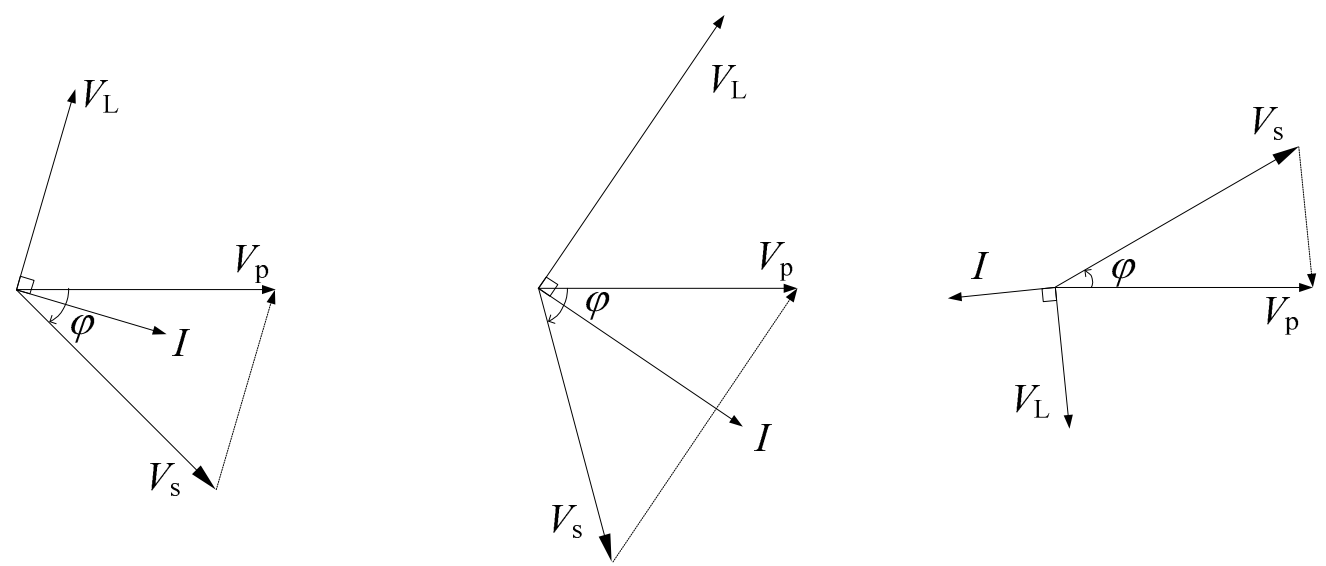

(a) power running mode (light load) (b) power running mode(heavy load) (c)power regenerative mode Fig. 3: Phasor diagram

\section{INTRINSIC SURGE PROBLEM OF A DAB DC-DC CONVERTER}

Well known problem of a DAB DC-DC converter is switching surge of when the load is in light condition. It is caused by the reverse recovery effect of the diode.

Figure 4 shows the switching surge generation mechanism when $V_{\text {in }}>V_{\text {out. }}$ The surge occurs in the transition from State 1 (3) to State 2 (4), repeatedly. $C_{\mathrm{d}}$ is the parasitic capacitance of diode which is connected in parallel with the ideal diode, and $L_{\text {wire }}$ is parasitic reactance. When the load is at the light load condition, the diodes $\mathrm{D}_{4}$ is conducting in State 1 . Then the switches $\mathrm{Q}_{3}$ is turned on when state changes from State 1 to State 2 . At this instantaneous moment, the diode $\mathrm{D}_{4}$ is switched from a forward bias condition to a reverse bias condition immediately, and it leads to resonance of $C_{\mathrm{d}}$ and $L_{\text {wire }}$. Because of these reasons, the switching surge occurs. Similarly, when $V_{\text {in }}<V_{\text {out }}$, the surge occurs in the transition from State 2 (4) to State 3 (1). it occurs in the bridge on the primary side.

Commonly, to protect the switches from the switching surge, snubber circuit are applied [2]. However, the power loss at the snubber circuit can't be ignored at the light load condition. The other way, the resonant converter type is also popular, but the additional components are needed.

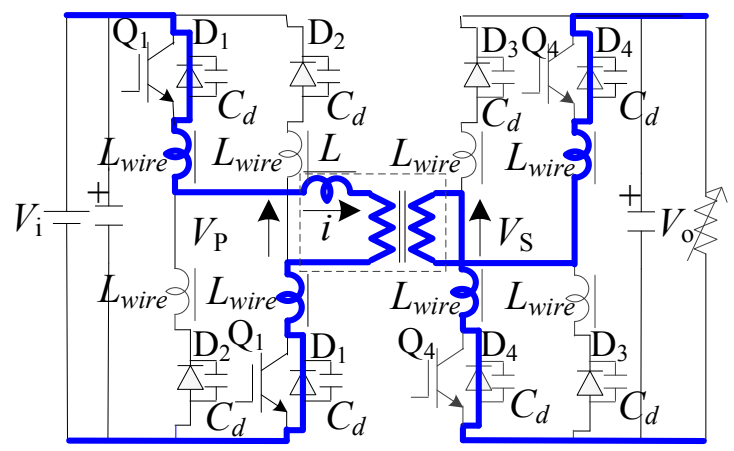

(a) State 1

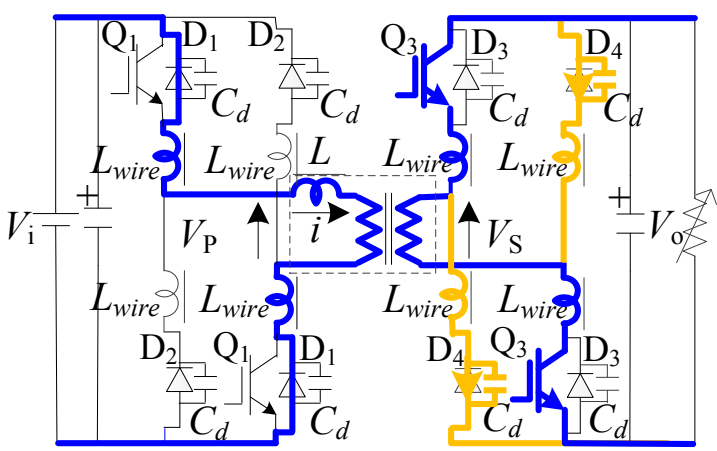

(b) State 2

Fig. 4: The switching surge generation mechanism: (a) State 1; (b) State 2

\section{PROPOSED OPERATING METHOD}

We propose the software-based compensation method for basic DAB bi-directional dc-dc converter which can be reduce the switching surge reduction at the light load, without any of additional circuits such as the snubber circuits or resonant circuits. Figure 5 shows idealized waveform of the proposed operating method. With this method, it can be easily change buck mode and boost mode operation.

When $V_{\text {in }}<V_{\text {out }}$, as it can be seen from the waveforms, the direction of primary side current of transformer i during each on-time of $\mathrm{Q}_{1}$ and $\mathrm{Q}_{2}$, is restricted to avoid the crossing the zero line. Due to the restriction of the change of the direction of the current, the zero-current-switching-on can be realized for $\mathrm{Q}_{1}$ and $\mathrm{Q}_{2}$. To analyze the characteristics of the circuit, Extended State-Space Averaging Method 
[5] is applied. The analysis has been done for each of buck mode and boost mode operation, respectively. This converter has six operational states in one switching period for each of the buck and boost mode operation, respectively. The elements are treated as ideal in equivalent circuit.

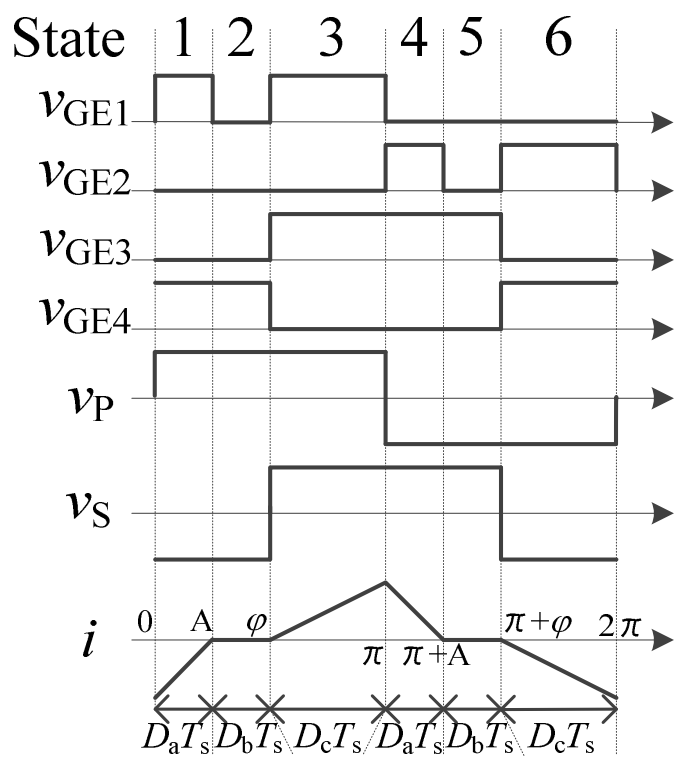

(a) buck mode

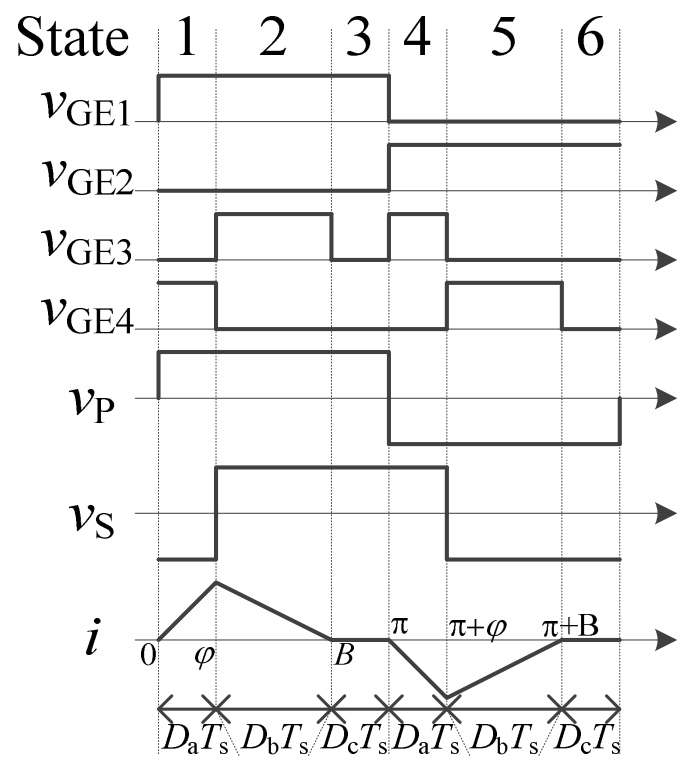

(b) boost mode

Fig. 5: Idealized wave form for proposed operating at the light load: (a) buck mode; (b) boost mode.

\section{Buck Mode Operation}

In buck mode, the primary side switches $\mathrm{Q}_{1}$ and $\mathrm{Q}_{2}$ are turned-on twice in the period. Firstly, $\mathrm{Q}_{1}$ and $\mathrm{Q}_{2}$ are turn-on at $t=0$ and $T_{\mathrm{S}} / 2$. Secondly, they are turn-off at $t=A$ and $T_{\mathrm{S}} / 2+A$. Thirdly, they are turn-on at $t=\varphi$ and $T \mathrm{~s} / 2+\varphi$. Fourthly, they are turn-off at $t=T_{\mathrm{s}} / 2$ and $T_{\mathrm{s}}$. Equivalent circuits corresponding to each state in buck mode operation are shown in Fig. 6, where $\hat{v}_{o}$ is the low-frequency component of $V_{\mathrm{o}}$. For analysis, solving for $i_{\mathrm{L}}$ and $i_{\mathrm{c}}$,

for $0 \leq t \leq A$ (State 1$)$

$$
i_{L}=\frac{V_{i}+\hat{v}_{0}}{L} t+i(0), \quad i_{C}=-i_{L}-\frac{\hat{v}_{o}}{R_{L}}
$$

for $A \leq t \leq \varphi$ (State 2)

$$
i_{L}=0, \quad i_{C}=-i_{L}-\frac{\hat{v}_{o}}{R_{L}}
$$

for $\varphi \leq t \leq \pi$ (State 3)

$$
i_{L}=\frac{V_{i}-\hat{v}_{0}}{L}\left\{t-\left(D_{a}+D_{b}\right) T_{s}\right\}, \quad i_{C}=i_{L}-\frac{\hat{v}_{o}}{R_{L}}
$$

for $\pi \leq t \leq \pi+A$ (State 4$)$

$$
i_{L}=-\frac{V_{i}+\hat{v}_{0}}{L}\left(t-\frac{1}{2} T_{s}\right)+\frac{V_{i}-\hat{v}_{0}}{L} D_{c} T_{s}, \quad i_{C}=i_{L}-\frac{\hat{v}_{o}}{R_{L}}
$$

for $\pi+A \leq t \leq \pi+\varphi$ (State 5)

$$
i_{L}=0, \quad i_{C}=i_{L}-\frac{\hat{v}_{o}}{R_{L}}
$$

for $\pi+\varphi \leq t \leq 2 \pi$ (State 6 )

$$
i_{L}=-\frac{V_{i}-\hat{v}_{0}}{L}\left\{t-\left(\frac{1}{2}+D_{a}+D_{b}\right) T_{s}\right\}, \quad i_{C}=-i_{L}-\frac{\hat{v}_{o}}{R_{L}} .
$$


From Fig. 5, it is clear that $D_{a}+D_{b}+D_{c}=1 / 2, i_{L}(0)=i_{L}(2 \pi)$ and $i_{L}(A)=0$. Using the preceding relationships,

$$
i_{L}(0)=i_{L}(2 \pi)=-\frac{V_{i}-\hat{v}_{0}}{L} D_{c} T_{s}
$$

and

$$
D_{c}=\frac{V_{i}+\hat{v}_{o}}{V_{i}-\hat{v}_{o}} D_{a}
$$

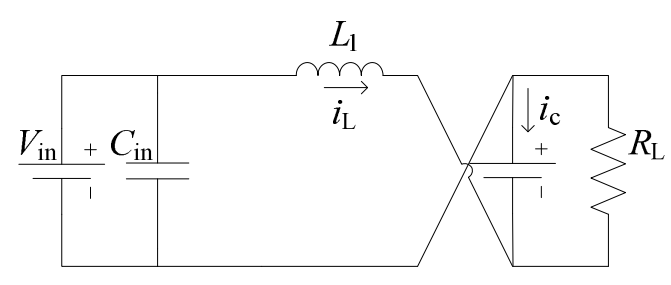

(a) State 1

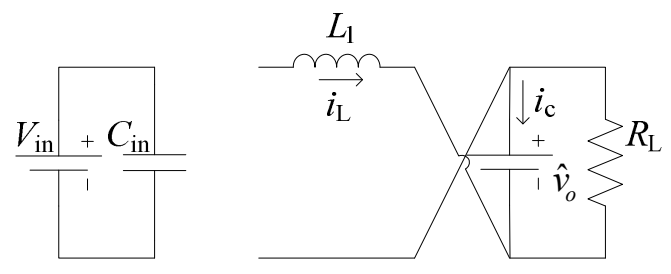

(b) State 2

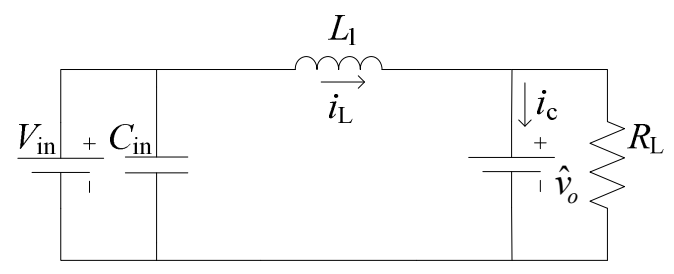

(c) State 3

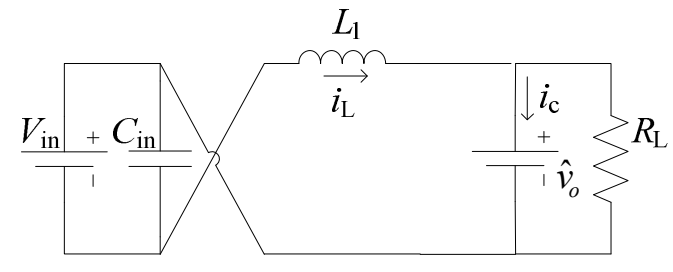

(d) State 4

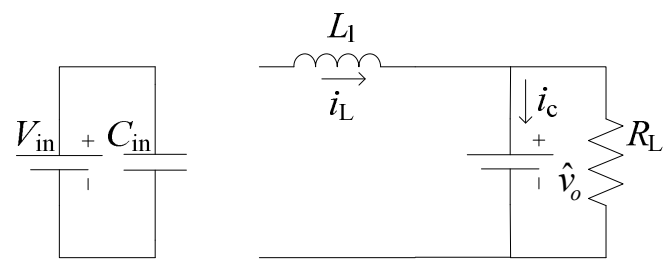

(e) State 5

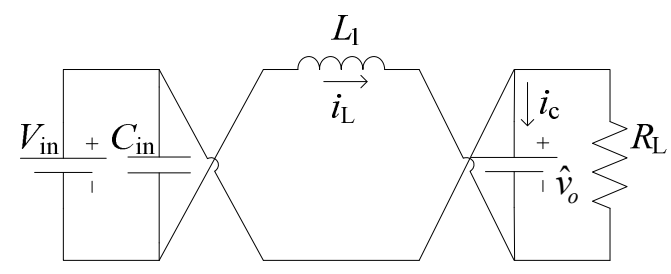

(f) State 6

Fig. 6 Equivalent circuit of buck mode operation: (a) State 1; (b) State 2; (c) State 3; (d) State 4; (e) State 5; (f) State 6

The average value of $i_{\mathrm{c}}$ in each state is calculated with

$$
\begin{aligned}
& i_{c_{-} \text {avel } 1}=\frac{1}{2}\left\{\left(\frac{V_{i}-\hat{v}_{o}}{L} D_{c} T_{s}-\frac{\hat{v}_{o}}{R_{L}}\right)-\frac{\hat{v}_{o}}{R_{L}}\right\}=\frac{1}{2} \frac{V_{i}-\hat{v}_{o}}{L} D_{c} T_{s}-\frac{\hat{v}_{o}}{R_{L}} \\
& i_{c_{-} \text {ave } 2}=-\frac{\hat{v}_{o}}{R_{L}} \\
& i_{c_{-} \text {ave } 3}=\frac{1}{2}\left\{-\frac{\hat{v}_{o}}{R_{L}}+\left(\frac{V_{i}-\hat{v}_{o}}{L} D_{c} T_{s}-\frac{\hat{v}_{o}}{R_{L}}\right)\right\}=\frac{1}{2} \frac{V_{i}-\hat{v}_{o}}{L} D_{c} T_{s}-\frac{\hat{v}_{o}}{R_{L}} \\
& i_{c_{-} \text {ave4 }}=\frac{1}{2}\left\{\left(\frac{V_{i}-\hat{v}_{o}}{L} D_{c} T_{s}-\frac{\hat{v}_{o}}{R_{L}}\right)-\frac{\hat{v}_{o}}{R_{L}}\right\}=\frac{1}{2} \frac{V_{i}-\hat{v}_{o}}{L} D_{c} T_{s}-\frac{\hat{v}_{o}}{R_{L}} \\
& i_{c_{-} \text {aves }}=-\frac{\hat{v}_{o}}{R_{L}} \\
& i_{c_{-} \text {ave }}=\frac{1}{2}\left\{-\frac{\hat{v}_{o}}{R_{L}}+\left(\frac{V_{i}-\hat{v}_{o}}{L} D_{c} T_{s}-\frac{\hat{v}_{o}}{R_{L}}\right)\right\}=\frac{1}{2} \frac{V_{i}-\hat{v}_{o}}{L} D_{c} T_{s}-\frac{\hat{v}_{o}}{R_{L}} .
\end{aligned}
$$

Hence,

$$
\overline{i_{c}}=i_{c_{-} a v e 1} \times D_{a}+i_{c_{-} a v e 2} \times D_{b}+i_{c_{-} a v e 3} \times D_{c}+i_{c_{-} a v e 4} \times D_{a}+i_{c_{-} a v e 5} \times D_{b}+i_{c_{-} a v e 6} \times D_{c}
$$




$$
=\frac{2 D_{a}^{2} T_{s} V_{i}}{L} \cdot \frac{V_{i}+\hat{v}_{o}}{V_{i}-\hat{v}_{o}}-\frac{\hat{v}_{o}}{R_{L}} .
$$

The results of static characteristics are obtained by letting $d \widehat{v}_{o} / d t=0$, therefore

$$
\begin{aligned}
& \frac{d \hat{v}_{o}}{d t}=\frac{1}{C} \overline{i_{c}} \\
& P_{o}=\frac{2 D_{a}^{2} T_{s}}{L} \cdot \frac{V_{o}+V_{i}}{V_{o}-V_{i}} \cdot V_{i} V_{o} .
\end{aligned}
$$

Using $D_{a} T_{s}=A$,

$$
P_{o}=\frac{2 A^{2}}{T_{s} L} \cdot \frac{V_{o}+V_{i}}{V_{o}-V_{i}} \cdot V_{i} V_{o}
$$

where

$$
A=\frac{V_{i}-V_{o}}{V_{i}+V_{o}}\left(\frac{1}{2} T_{s}-\varphi\right) .
$$

\section{Boost Mode Operation}

In boost mode, the secondary side switches $\mathrm{Q}_{3}$ and $\mathrm{Q}_{4}$ are turned-on twice in the period. Firstly, $\mathrm{Q}_{3}$ and $\mathrm{Q}_{4}$ are turn-on at $t=\varphi$ and $T_{\mathrm{s}} / 2+\varphi$, respectively. Secondly, they are turn-off at $t=B$ and $T_{\mathrm{s}} / 2+$ $B$. Thirdly, they are turn-on at $t=T_{\mathrm{s}} / 2$ and 0 . Fourthly, they are turn-off at $t=T_{\mathrm{S}} / 2+\varphi$ and $B$. Equivalent circuits corresponding to each state in boost mode operation are shown in Fig. 7.

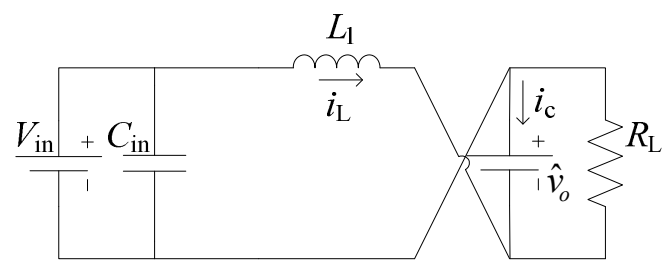

(a) State 1

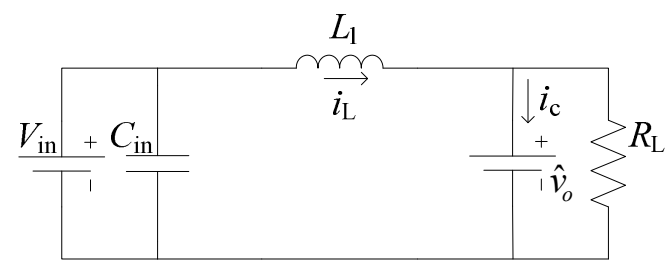

(b) State 2

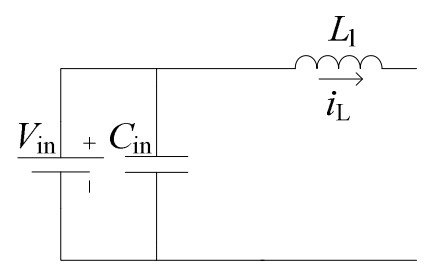

(c) State 3

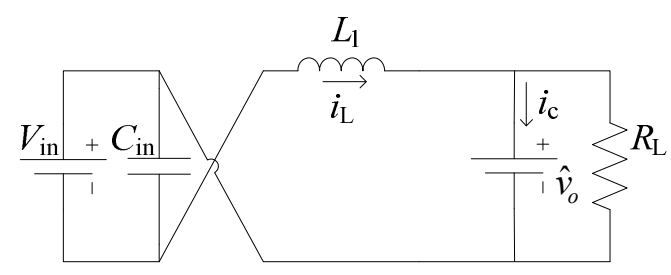

(d) State 4

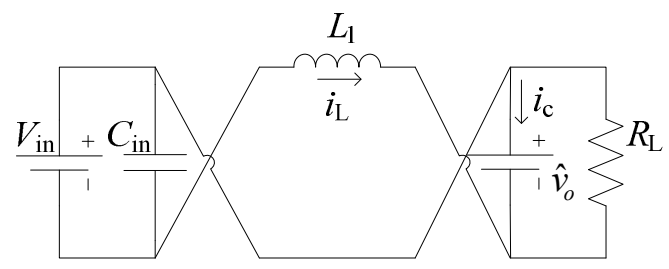

(e) State 5

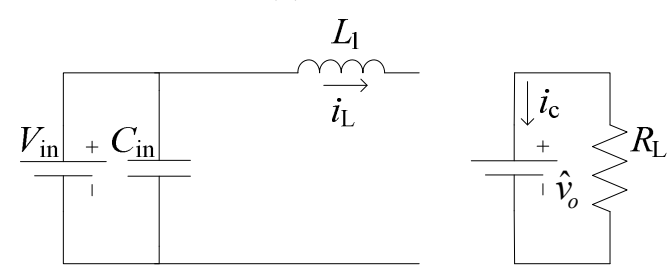

(f) State 6

Fig. 7 Equivalent circuit of boost mode operation: (a) State 1; (b) State 2; (c) State 3; (d) State 4;

(e) State 5; (f) State 6

For analysis, equation is formularized for each state.

For $0 \leq t \leq \varphi($ State 1$)$

$$
i_{L}=\frac{V_{i}+\hat{v}_{0}}{L} t, \quad i_{C}=-i_{L}-\frac{\hat{v}_{o}}{R_{L}},
$$

for $\varphi \leq t \leq B$ (State 2) 


$$
i_{L}=\frac{V_{i}-\hat{v}_{0}}{L}\left(t-D_{a} T_{s}\right)+\frac{V_{i}+\hat{v}_{o}}{L} D_{a} T_{s}, \quad i_{C}=i_{L}-\frac{\hat{v}_{o}}{R_{L}},
$$

for $B \leq t \leq \pi$ (State 3)

$$
i_{L}=0, \quad i_{C}=-\frac{\hat{v}_{o}}{R_{L}}
$$

for $\pi \leq t \leq \pi+\varphi$ (State 4$)$

$$
i_{L}=-\frac{V_{i}+\hat{v}_{0}}{L}\left(t-\frac{1}{2} T_{s}\right), \quad i_{C}=i_{L}-\frac{\hat{v}_{o}}{R_{L}}
$$

for $\pi+\varphi \leq t \leq \pi+B$ (State 5)

$$
i_{L}=-\frac{V_{i}-\hat{v}_{0}}{L}\left\{t-\left(\frac{1}{2}+D_{a}\right) T_{s}\right\}-\frac{V_{i}+\hat{v}_{o}}{L} D_{a} T_{s}, \quad i_{C}=-i_{L}-\frac{\hat{v}_{o}}{R_{L}},
$$

for $\pi+B \leq t \leq 2 \pi$ (State 6 )

$$
i_{L}=0, \quad i_{C}=-\frac{\hat{v}_{o}}{R_{L}} .
$$

From Fig. 5, it is clear that $D_{a}+D_{b}+D_{c}=1 / 2$, and $i_{L}(B)=0$. Using the preceding relationships

$$
D_{b}=-\frac{V_{i}+\hat{v}_{o}}{V_{i}-\hat{v}_{o}} D_{a}
$$

The average value of $i_{\mathrm{c}}$ in each state is calculated with

$$
\begin{aligned}
& i_{c_{-} \text {ave } 1}=\frac{1}{2}\left\{-\frac{\hat{v}_{o}}{R_{L}}+\left(-\frac{V_{i}+\hat{v}_{o}}{L} D_{a} T_{s}-\frac{\hat{v}_{o}}{R_{L}}\right)\right\}=-\frac{1}{2} \frac{V_{i}+\hat{v}_{o}}{L} D_{a} T_{s}-\frac{\hat{v}_{o}}{R_{L}} \\
& i_{c_{-} \text {ave } 2}=\frac{1}{2}\left\{\left(\frac{V_{i}+\hat{v}_{o}}{L} D_{a} T_{s}-\frac{\hat{v}_{o}}{R_{L}}\right)-\frac{\hat{v}_{o}}{R_{L}}\right\}=\frac{1}{2} \frac{V_{i}+\hat{v}_{o}}{L} D_{a} T_{s}-\frac{\hat{v}_{o}}{R_{L}} \\
& i_{c_{-} \text {ave3 }}=-\frac{\hat{v}_{o}}{R_{L}} \\
& i_{c_{-} \text {ave4 }}=\frac{1}{2}\left\{-\frac{\hat{v}_{o}}{R_{L}}+\left(-\frac{V_{i}+\hat{v}_{o}}{L} D_{a} T_{s}-\frac{\hat{v}_{o}}{R_{L}}\right)\right\}=-\frac{1}{2} \frac{V_{i}+\hat{v}_{o}}{L} D_{a} T_{s}-\frac{\hat{v}_{o}}{R_{L}} \\
& i_{c_{-} \text {ave } 5}=\frac{1}{2}\left\{\left(\frac{V_{i}+\hat{v}_{o}}{L} D_{a} T_{s}-\frac{\hat{v}_{o}}{R_{L}}\right)-\frac{\hat{v}_{o}}{R_{L}}\right\}=\frac{1}{2} \frac{V_{i}+\hat{v}_{o}}{L} D_{a} T_{s}-\frac{\hat{v}_{o}}{R_{L}} \\
& i_{c_{-} \text {ave6 }}=-\frac{\hat{v}_{o}}{R_{L}} .
\end{aligned}
$$

Hence,

$$
\overline{i_{c}}=-\frac{2 D_{a}^{2} T_{s} V_{i}}{L} \cdot \frac{V_{i}+\hat{v}_{o}}{V_{i}-\hat{v}_{o}}-\frac{\hat{v}_{o}}{R_{L}} .
$$

The results of static characteristics are obtained by letting $d \hat{v}_{o} / d t=0$, therefore

$$
P_{o}=\frac{2 D_{a}^{2} T_{s}}{L} \cdot \frac{V_{o}+V_{i}}{V_{o}-V_{i}} \cdot V_{i} V_{o} .
$$

Using $D_{a} T_{s}=\varphi$

$$
P_{o}=\frac{2 \varphi^{2}}{T_{s} L} \cdot \frac{V_{o}+V_{i}}{V_{o}-V_{i}} \cdot V_{i} V_{o} .
$$

$B$ is calculated as 


$$
B=\left(D_{a}+D_{b}\right) T_{s}=\frac{2 V_{o}}{V_{o}-V_{i}} \varphi .
$$

\section{Output Power}

From the above, the analysis for both of buck and boost mode operation can be done in uniformly. For the analysis result, the output power Po can be obtained as

$$
P_{o}=\frac{2 X^{2}}{T_{s} L} \cdot\left|\frac{V_{i}+V_{o}}{V_{i}-V_{o}}\right| \cdot V_{i} V_{o} .
$$

In buck mode, $X=A$, and in boost mode, $X=\varphi$.

\section{Operation Shift between Light and Heavy Load}

In the light load, with the output power increasing, the periods of which all switches turned OFF ( $A \sim \varphi, \pi+A \sim \pi+\varphi, B \sim \pi, \pi+B \sim 2 \pi$ ) becomes shorter. The periods, equal to zero seconds, it is the boundary between light load and heavy load. Therefore, in the heavy load condition, the only conventional phase-shift operation is active. From the results, it can be seen that it is possible to control the output power seamlessly despite of the load condition.

\section{EXPERIMENTAL RESULTS}

To verify the proposed operation validity, we perform some experiments with the prototype circuit. The main circuit is DAB dc-dc converter without additional circuits like snubber circuit. We had closed loop operation experiments with DSP TI TMS320F28335. Experimental parameters are shown in Table I. Figure 8 and Fig. 9 show drive signal generating mechanism. Dead time of each switch is $1 \mu$ s.

Table I: Specification of Bidirectional DAB dc-dc converter

\begin{tabular}{|l|l|l|}
\hline \multicolumn{1}{|c|}{ Item } & Symbol & Specification \\
\hline Transformer & & \\
\hline 1) Turns ratio & $a$ & $1: 1$ \\
\hline 2) Leakage inductance(primary-referred) & $L$ & $110 \mu \mathrm{H}$ \\
\hline Converter & & \\
\hline 1) Rated output power & $P_{\mathrm{o}}$ & $1 \mathrm{~kW}$ \\
\hline 2) Rated input direct voltage & $V_{\mathrm{in}}$ & $150 \mathrm{~V}$ \\
\hline 3) Rated output direct voltage & $V_{\text {out }}$ & $150 \mathrm{~V}$ \\
\hline 4) Switching frequency & $f_{\mathrm{s}}$ & $20 \mathrm{kHz}$ \\
\hline 5) Absolute maximum ratings of IGBT collector-emitter & $v_{\mathrm{CE}}$ & $600 \mathrm{~V}$ \\
\hline 6) On resistance of IGBT & $r_{\mathrm{t}}$ & $50 \mathrm{~m} \Omega$ \\
\hline 7) Absolute maximum ratings of diode & $i_{\mathrm{F}}$ & $30 \mathrm{~A}$ \\
\hline 8) Forward voltage of diode & $v_{\mathrm{F}}$ & $0.8 \mathrm{~V}$ \\
\hline 9) Recovery time of diode & $t_{\mathrm{rr}}$ & $0.1 \mu \mathrm{s}$ \\
\hline
\end{tabular}

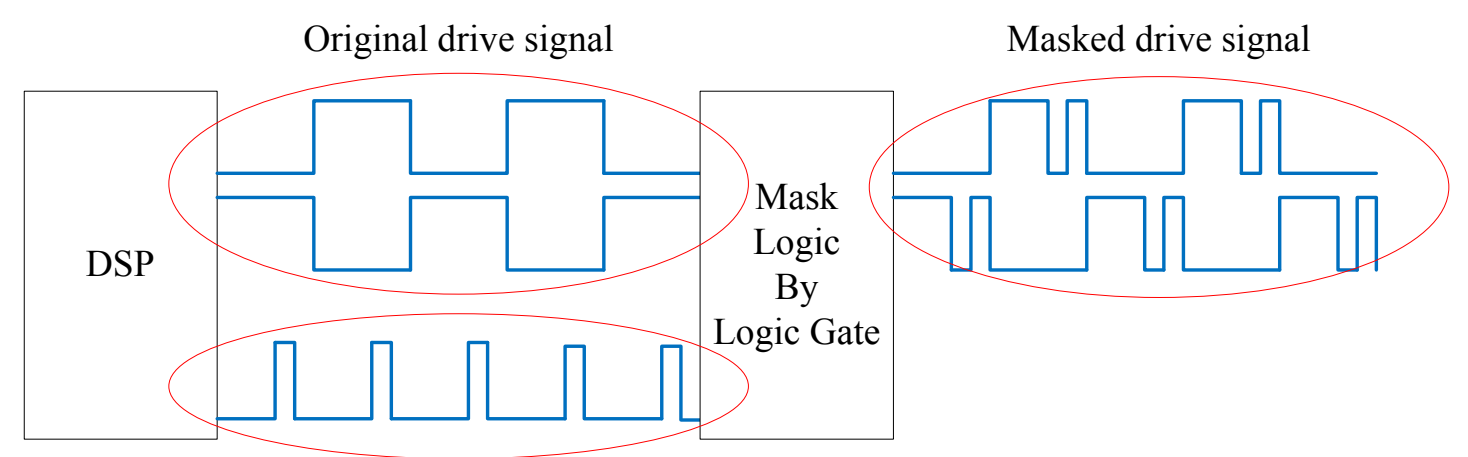

Mask signal

Fig. 8 Masked drive signal generating mechanism. 


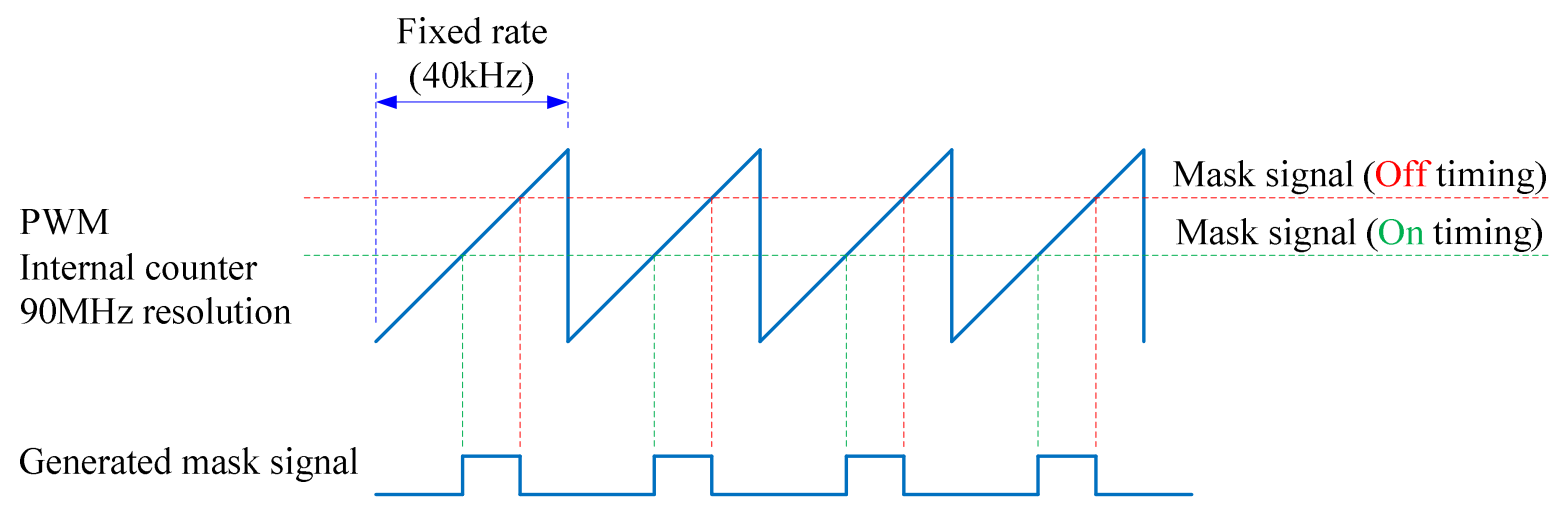

Fig. 9 Mask signal generating mechanism by PWM peripheral in DSP.

Figure 10 shows the waveform of the corrector-emitter voltage and the corrector current of the low voltage side bridge of the buck converter. Figure 10(a) shows the result of the conventional operation and Figure 10(b) shows the result of the proposed operation. Comparing with these results, it can be seen that $99 \%$ of voltage surges and $100 \%$ of current serge of reduction.

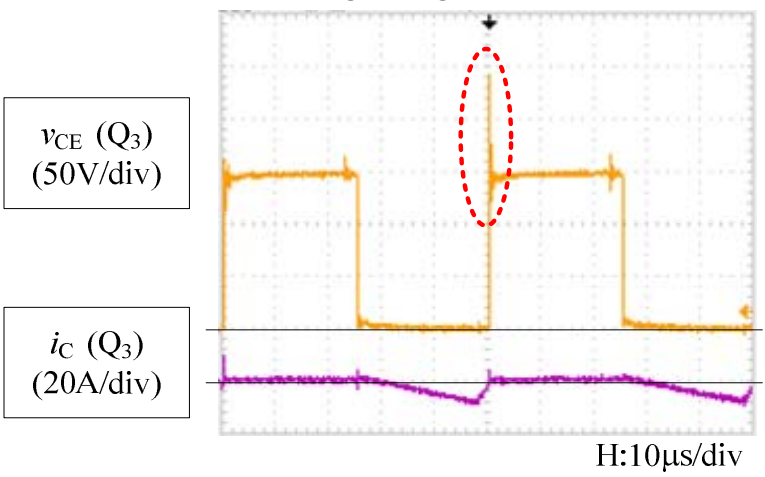

(a) the prototype operation

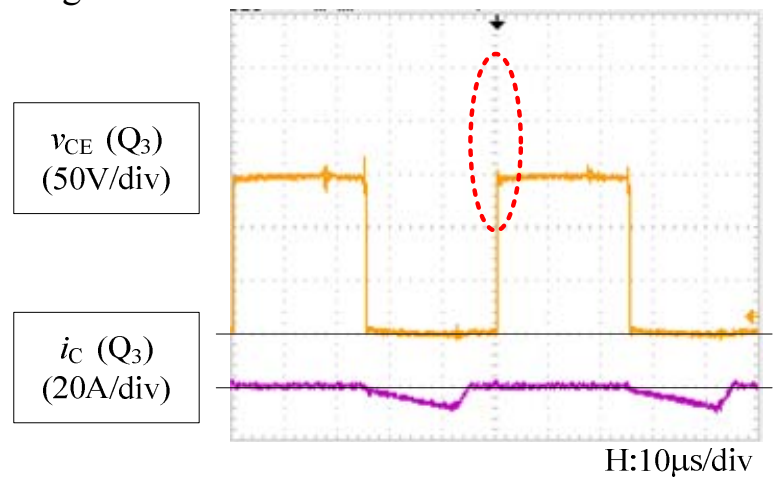

(b) the proposed operation

Fig. 10 The waveforms in buck mode: (a) the prototype operation $\left(V_{\text {in }}=200 \mathrm{~V}, V_{\text {out }}=150 \mathrm{~V}, P_{\mathrm{o}}=569 \mathrm{~W}\right)$;

(b) the proposed operation. $\left(V_{\text {in }}=200 \mathrm{~V}, V_{\text {out }}=150 \mathrm{~V}, P_{\mathrm{o}}=566 \mathrm{~W}\right)$

Figure 11 shows the waveform of the boost converter. Comparing with these results, it can be seen that $99 \%$ of voltage surges of reduction and $100 \%$ of current serge of reduction.

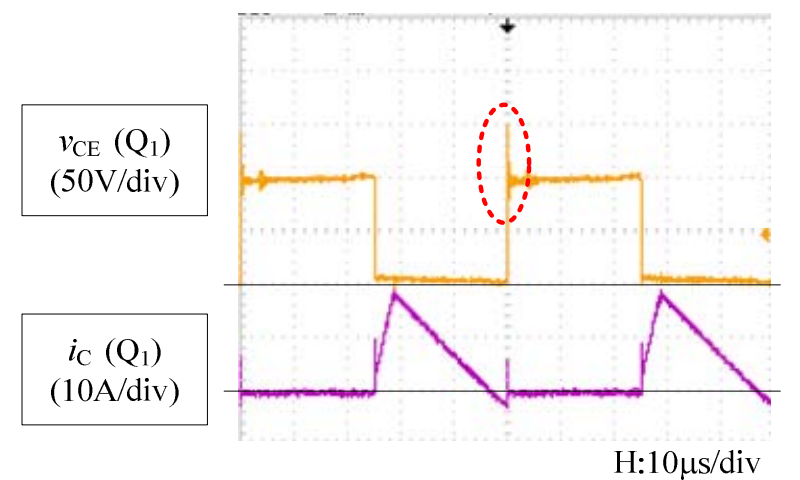

(a) the prototype operation

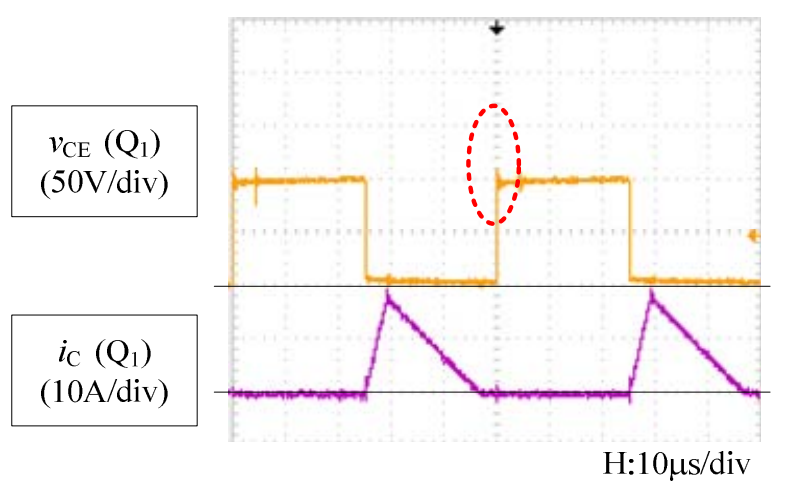

(b) the proposed operation

Fig. 11 The waveforms in boost mode: (a) the prototype operation $\left(V_{\text {in }}=100 \mathrm{~V}, V_{\text {out }}=150 \mathrm{~V}, P_{\mathrm{o}}=687 \mathrm{~W}\right)$; (b) the proposed operation. $\left(V_{\text {in }}=100 \mathrm{~V}, V_{\text {out }}=150 \mathrm{~V}, P_{\mathrm{o}}=686 \mathrm{~W}\right)$

Figure 12 shows the power efficiency results for the both of the conventional and the proposed operation. It can be seen that the power efficiency of buck mode can be apparently improved by up to $37 \%$ using the proposed operation at 100W as shown in Fig. 12 (a). It can be seen that the power efficiency of boost mode can be apparently improved by up to $30 \%$ at $100 \mathrm{~W}$ as shown in Fig. 12 (b). 


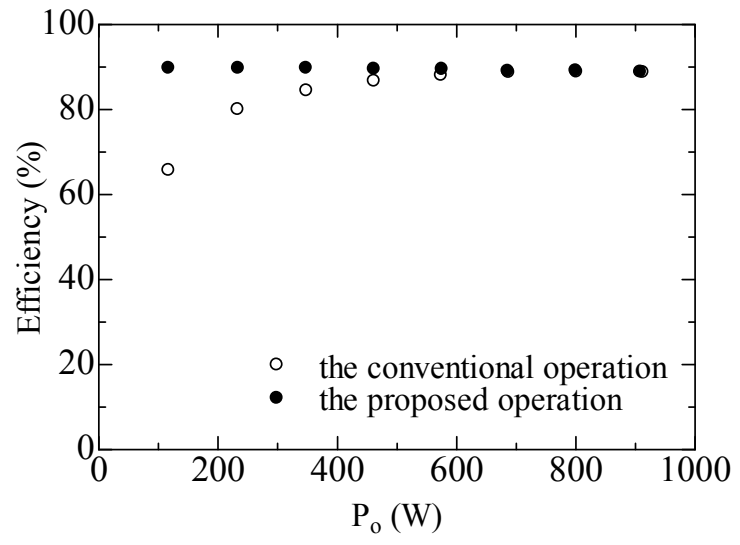

(a) buck mode

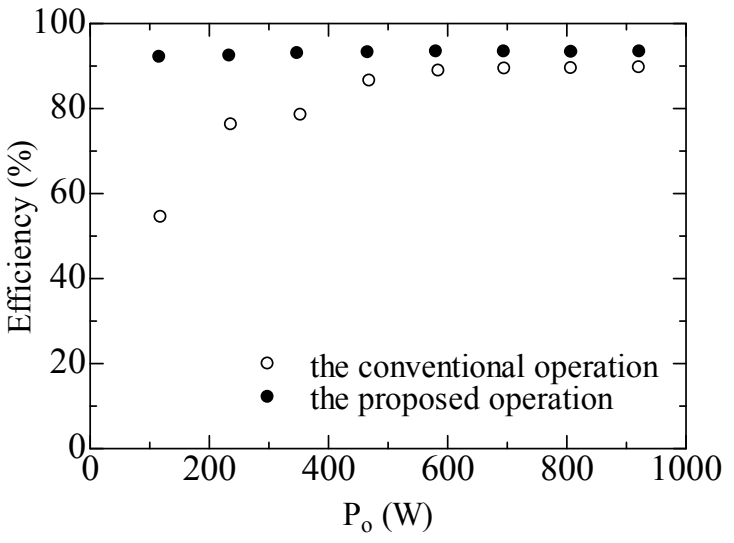

(b) boost mode

Fig. 12 Power efficiency: (a) buck mode $\left(V_{\text {in }}=200 \mathrm{~V}, V_{\text {out }}=150 \mathrm{~V}\right)$;

(b) boost mode $\left(V_{\text {in }}=100 \mathrm{~V}, V_{\text {out }}=150 \mathrm{~V}\right)$

\section{Conclusion}

By the analysis of the circuit operation and the some experiments, the validation of the proposed operation for DAB dc-to-dc converter is revealed. The operation method with digital operation can reduce switching surges without other circuits like snubber circuit. From the experiment results, the $99 \%$ of the maximum voltage surge reduction and $100 \%$ of the maximum current serge reduction at the light load is confirmed. Furthermore, due to the surge reduction method, $37 \%$ maximum power efficiency improvement can be confirmed at light load. Also the operation can be applied to any condition from light to heave load seamlessly.

\section{References}

[1] Mustansir H. Kheraluwala, Randal W. Gascoigne, Deepakraj M. Divan, and Eric D. Baumann, "Performance characterization of a high-power dual active bridge dc-to-dc converter," IEEE Trans. Industry Applications, vol.28, NO.6, pp. 1294-1301, Nov. / Dec. 1992

[2] Shigenori Inoue and Hirofumi Akagi, "A bidirectional isolated dc-dc converter as a core circuit of the nextgeneration medium-voltage power conversion system,” IEEE Trans power Electron., vol.22, no.2, pp. 535-542, Mar. 2007.

[3] M. Pavlovsky, S. W. H. de Hann, and J. A. Ferreira, "Concept of 50kW DC/DC converter based on ZVS, quasi-ZCS topology and integrated thermal and electronic design," 2005 European Conference on Power Electronics and Applications.

[4] Toshiro Hirose, Keisuke Nishimura, Takayuki Kimura, and Hirofumi Matsuo, "An AC-link Bidirectional DC-DC Converter with Synchronous Rectifier," in Proc. IECON, Nov. 2010.

[5] Rik W. A. A. De Doncker, Deepkraj M. Divan, and Mustansir H. Kheraluwala, "A Three-Phase SoftSwitched High-Power-Density dc/dc Converter for High-Power Applications," IEEE Trans. Industry Applications, vol.27, NO.1, pp.63-73, Jan. / Feb. 1991

[6] Tamotsu Ninomiya, Masatoshi Nakahara, Toru Higashi, and Koosuke Harada, "A Unified Analysis of resonant Converters," IEEE Trans. Industry Applications, vol.27, NO.1, pp.63-73, Jan. / Feb. 1991 